\section{Why genes can be patented}

SIR - Fergus Davison highlights a difficult point in the understanding of patents derived from genetic studies (Nature 379, 111; 1996). I, too, thought George Poste (Nature 378, 534-536; 1995) skipped over the distinction between discoveries and inventions.

Perhaps some enlightenment can be given by reference to another well known example. Antibiotics have been patented for years without the challenges that have been applied to DNA sequences, but they are also natural molecules produced by living organisms and are, in a very real sense, discovered. Why, then, are they classed as inventions and therefore patentable?

The fact is that the antibiotics produced by living organisms in their wild state are not patentable. However, if an individual or a company searches for and finds the right organism, identifies a useful property of one of its metabolites, isolates, purifies and characterizes the product and devises a method of making and using it, then the outcome is not the antibiotic in its natural state but the genuine product of human ingenuity. It is therefore classed as an invention. The route to finding new antibiotics was used many times over, but the novel chemicals that emerged remained patentable. It is greatly to our advantage that this has been and continues to be the case. Discovering and developing new antibiotics, as with all drugs, is an expensive process, and the patent system is a major mechanism whereby society can encourage scientists in or outside companies to search for new remedies for the general good.

The same arguments apply to DNA sequences. In their natural state, within the body of an organism, directing the production of enzymes, hormones or whatever, they are not patentable. But a DNA sequence that can specify erythropoietin, for example, when identified, characterized and transferred to a suitable production, can give a product that, when purified and properly formulated, is of great value to some patients. In this process, neither erythropoietin nor its gene is in its natural state, and the DNA sequence, as a component of the system that can make such a useful product, is justifiably patentable. Some of the techniques might seem routine to an expert in gene cloning, as would some of the steps in identifying an antibiotic to a microbial ecologist, but the tasks that face Poste and his colleagues are far from trivial, and if society wants new medicines it had better make sure that someone is encouraged to discover them and make them available.

On the arguments given above, Newton could not have patented gravity any more than someone who had just discovered its secrets could patent the electrical phenomena that comprise the lightning of an electrical storm. However, if someone could take components of those phenomena and reproduce them in a laboratory in a controlled way and then show how they could be used, should there not be a mechanism for ensuring that that person is recompensed for the benefit that accrues to society? Those who have difficulties with DNA patents should consider that question each time they turn on an electric light.

\section{Norman H. Carey}

Russells Close, High Street,

Chinnor, Oxfordshire OX9 4DJ, UK

\section{Chance remarks}

SIR - Every week, millions of British people buy national lottery tickets, all hoping to be the one who accurately predicts 6 numbers lying between 1 and 50 . Every other week or so, someone succeeds. The winner is not clairvoyant, merely living proof that if enough trials are performed, even extraordinarily unlikely events may be observed. Along similar lines, most scientists know that a table containing 20 independent statistical tests for a non-existent trend will, on average, yield one value indicating significance with $P<0.05$. Such a value is referred to as a 'type I error', and should properly be ignored.

However, it is interesting to go beyond a single paper to look at how type I errors might operate between laboratories. Consider the fictitious fate of 20 behavioural ecologists who encounter the same provocatively stimulating dataset at a seminar given by a colleague. All 20 privately develop the same exciting hunch which they then test independently, each with a single experiment. Even if the common hunch has no basis, the odds favour one of these scientists finding a 'significant' value at the $P<0.05$ level, which might get published.

Following publication, 1,000 desperate lecturers searching for interesting undergraduate projects all opt for a simple oneexperiment test of this new finding. The projects are completed and 50 yield significant values at the 0.05 level, 10 reach 0.01 and one even attains 0.001 . With a bird's-eye view, a neutral observer would realise that there all these 'significant' values are likely to be type I errors. However, from the point of view of the individual, there are now 50 excited supervisors of whom perhaps 30 prepare for immediate publication.

Now, suppose the remaining 20 lecturers are more cautious and tell their students to repeat the experiment. One person manages to reproduce his or her results and publishes a convincing paper, whilst the others shake their heads and ascribe their fluctuating fortunes to student error. Of course, the bird's-eye observer is still not surprised: there are 20 new tests and one yields a $P<0.05$. Of course, the happy 'winner' is blissfully unaware that the result is a type I error, and the scientific community, faced with a widely publicized seminal paper followed swiftly by 31 pieces of supporting evidence, one of which appears really quite convincing, now believes the trend to be real. Yet it never existed.

Finally, we should bear in mind that experiments do not have to be related. Most statistical tests are essentially equivalent, and ask simply whether there has been a significant deviation from random. All over the world, vast numbers of such tests are performed daily, spread across many disciplines. Where trends exist, they will tend to be detected. However, among the vast number of tests applied to nonexistent trends, many spuriously significant $P$-values will arise, some of which will be extreme. The exact number of artefactual findings will be related to the number of tests that 'fail', which is never likely to be determined. Consequently, it is perhaps worth bearing in mind that there is such a thing as a global type I error, and that individually good statistical practice does not necessarily remove the risk.

\section{Bill Amos}

University of Cambridge,

Department of Genetics,

Downing Street,

Cambridge CB2 3EH, UK

\section{Well reviewed}

SIR - As one of the lead authors of the Intergovernmental Panel on Climate Change (IPCC) 1994 Scientific Assessment, I must protest about Richard Courtney's ridiculous claim (Nature 379, 109; 1995) that "IPCC approved the summary of its 1994 Scientific Assessment before that report was written".

The summary of the 1994 report was approved in Maastricht in September 1994. By that time, the report was virtually complete, having undergone, first, peer review in February-April 1994 and, after modification, a review by each country during May-July 1994.

This review process was not a minor one - it entailed hundreds of copies of the drafts being sent out for review. Indeed, I would be very surprised to learn, given $\mathrm{Mr}$ Courtney's interest in the IPCC process, that he had not seen at least one of these earlier drafts.

\section{Keith P. Shine}

Department of Meteorology,

University of Reading,

2 Earley Gate,

Reading RG6 2AU, UK 